\title{
Necesidades de salud mental en adolescentes en conflicto con la ley penal: Un estudio descriptivo en una muestra de Argentina
}

\author{
Karin Arbach ${ }^{1}$, Cinderella Orpianesi² y Antonella Bobbio ${ }^{3}$
}

\begin{abstract}
RESUMEN
Los adolescentes en conflicto con la ley penal presentan más necesidades de salud mental que los de población general. El cribado de estas necesidades en instancias de admisión a los dispositivos judiciales permite identificar aquellos casos que requieren una intervención específica e inmediata. El presente estudio explora la prevalencia y comorbilidad de necesidades de salud mental en 55 adolescentes admitidos en un centro cerrado del sistema de justicia juvenil mediante una versión traducida para Argentina del MAYSI-2 (Grisso, Barnum, Orpianessi \& Arbach, 2018). Aproximadamente el 90\% de los adolescentes presentaron indicadores de al menos un tipo de necesidad de salud mental, y una proporción similar indicó haber vivenciado experiencias traumáticas a lo largo de su vida. Las condiciones clínicas más prevalentes fueron la somatización, la perturbación del pensamiento y los síntomas depresivos y ansiosos. La subescala de abuso de alcohol y drogas fue la que registró la mayor proporción de adolescentes con puntuaciones por encima del máximo punto de corte. Casi la totalidad de adolescentes presentaron indicadores de dos o más condiciones comórbidas. Se discuten las implicancias prácticas de estos hallazgos y la importancia de implementar estrategias de cribado en contextos de recursos limitados y donde el MAYSI-2 podría resultar una herramienta prometedora.
\end{abstract}

Palabras clave: salud mental, MAYSI2, adolescentes, justicia penal.

\section{Mental health needs in juvenile offenders: A descriptive study in an Argentinean sample}

\begin{abstract}
Juveniles in the criminal justice system have higher rates of mental health needs than those in the general population. The screening of these needs in admission to the legal devices allows identifying those cases that require specific and immediate intervention. The present study explores the prevalence and comorbidity of mental health needs in 55 adolescents admitted to a closed detention center of the juvenile justice system through a translated version for MAYSI-2 for Argentina (Grisso, Barnum, Orpianessi \& Arbach, 2018). Approximately $90 \%$ of the adolescents presented scores that indicated the probable presence of at least one type of mental health need. A similar proportion reports having experienced traumatic experiences along their lives. The most prevalent clinical conditions were somatization, thought disturbance, and depressive and anxious
\end{abstract}

1 Universidad Nacional de Córdoba, Consejo Nacional de Investigaciones Científicas y Tecnológicas, Argentina; k_arbach@hotmail.com; http://orcid.org/0000-0003-1753-4693

2 Universidad Nacional de Córdoba, Argentina; cinderella.orpianesi@gmail.com; http://orcid.org/00000002-6933-8865

3 Universidad Nacional de Córdoba, Consejo Nacional de Investigaciones Científicas y Tecnológicas, Argentina; antonellabobbio@hotmail.com; https://orcid.org/0000-0003-4121-9482

AGRADECIMIENTOS | Al Dr. Thomas Grisso por su contribución en la traducción de la versión argentina del MAYSI-2 y sus comentarios sobre este manuscrito. A las autoridades institucionales y los participantes. 
symptoms. The alcohol and drug abuse subscale recorded the highest proportion of adolescents with scores above the maximum cut-off point. Almost all of the cases had scores that indicated the presence of two or more comorbid conditions. MAYSI- 2 could be a promissory tool in the implementation of screening strategies in resource-limited settings. Practical and research implications of these findings are discussed.

Keywords: mental health needs, MAYSI2, adolescents, criminal justice.

Cada año aproximadamente 4000 adolescentes menores de 18 años ingresan al sistema de justicia penal juvenil de Argentina y cerca del $20 \%$ de ellos son alojados en dispositivos de privación de su libertad (UNICEF, 2015). De estos últimos, cerca de la mitad requerirá atención psiquiátrica o psicológica tras su ingreso (UNICEF, 2018). Córdoba es una de las provincias en ese país con las tasas más altas de jóvenes detenidos. De los 1200 adolescentes evaluados cada año por la presunta comisión de delitos, aproximadamente $65 \%$ son derivados a un régimen de internamiento cerrado (Tribunal Superior de Justicia, 2016). Estos hechos, sumados a las barreras en la accesibilidad al sistema de salud que enfrentan los grupos más vulnerables de adolescentes en el medio comunitario (UNICEF, 2016), requieren al sistema penal juvenil generar estrategias eficaces para valorar y asistir las necesidades de salud mental que puedan presentar los adolescentes que alberga.

Lo anterior cobra especial relevancia ante los hallazgos que muestran que los jóvenes en conflicto con la ley penal presentan una prevalencia mayor de problemas de salud mental comparados con los jóvenes de población general (Bailey, Doreleijers, \& Tarbuck, 2006; Fazel, Doll, \& Långström, 2008; Penner, Roesch, \& Viljoen, 2011). Una revisión de 15 estudios de 10 países de altos ingresos que analizó una muestra total de 3400 adolescentes en el sistema de justicia penal encontró que el $70 \%$ cumplia criterios diagnósticos para al menos un trastorno mental, en comparación con el $16 \%$ de la población general (Colins, Vermeiren, Vreugdenhil, Doreleijers, \& Broekaert, 2010). Geográficamente más cercano, un estudio en Chile encontró que el $84 \%$ de 489 adolescentes en un centro de detención cumplía criterios diagnósticos para algún problema de salud mental (Gaete, Labbé, del Villar, Allende, \& Valenzuela, 2014). Este dato contrasta con una prevalencia de problemas de salud mental cercana al $23 \%$ en población general de ese rango etario en ese país (De La Barra, Vicente, Saldivia, \& Melipillán, 2012).

En términos generales, los problemas de salud mental más prevalentes en los adolescentes en conflicto con la ley se vinculan a los diagnósticos de trastornos de conducta y por consumo de sustancias, seguidos por los trastornos por hiperactividad y déficit de atención, y los trastornos del estado de ánimo y de ansiedad (Colins et al., 2010; Gaete et al., 2014; Grisso, Vincent, \& Seagrave, 2005; Livanou, Furtado, Winsper, Silvester, \& Singh, 2019; Schubert, Mulvey, \& Glasheen, 2011; Schufelt \& Cocozza, 2006). En relación con los trastornos mentales graves, una revisión sistemática de 25 estudios encontró que los adolescentes ingresados en centros de detención tienen 10 veces más probabilidades de presentar algún diagnóstico de psicosis comparados con los jóvenes de la población general (Fazel et al., 2008).

También en estos adolescentes se han reportado tasas elevadas de ideación y conductas suicidas y autolesivas que oscilan entre el $10 \%$ y el $60 \%$ 
(Bhatta et al., 2014; Moore, Gaskin, \& Indig, 2015), que se han mostrado superiores a las de jóvenes de población general (Hayes, 2009; Scott, Underwood, \& Lamis, 2015). Estas formas de violencia autodirigida se han encontrado asociadas a experiencias traumáticas y de victimización (Finkelhor, Ormrod, \& Turner, 2007; Ford, Hartman, Hawke, \& Chapman, 2008) y a la presencia de trastorno por estrés post-traumático (Abram et al., 2004). Por ejemplo, un estudio español encontró en una muestra de 100 adolescentes ingresados en el sistema de justicia juvenil que todos reportaban haber sufrido al menos un tipo de victimización interpersonal y un promedio de 10 victimizaciones a lo largo de la vida (Suárez-Soto, Pereda, \& Guilera, 2018).

Los diferentes problemas de salud mental identificados en esta población presentan una alta comorbilidad (Abram et al., 2015). Este hecho agrava la evaluación y atención psicológicas en general, y la que se ofrece en el sistema de justicia penal en particular. Por ejemplo, un estudio con una muestra total de 1400 adolescentes de ambos sexos residentes en un centro del sistema penal juvenil de Estados Unidos encontró que aproximadamente el $80 \%$ cumplía criterios para dos o más diagnósticos simultáneos, y que en más de la mitad de los casos la condición comórbida era el abuso de sustancias (Schufelt \& Cocozza, 2006). Estos hallazgos indican que el énfasis no debería estar en indagar de manera exclusiva sobre un problema específico y focalizado, sino que es indispensable reconocer la existencia simultánea de múltiples dificultades relacionadas con la salud mental de estos adolescentes (Grisso et al., 2005).

El reconocimiento de la alta prevalencia de las necesidades de salud mental en la población juvenil judicializada condujo al desarrollo de técnicas de detección rigurosas y programas de atención de salud mental en los contextos penales juveniles de distintos países del mundo (Schubert et al., 2011). La Academia Americana de Psiquiatría Infantil y Adolescente (American Academy of Child and Adolescent Psychiatry; www.aacap.org/) sugiere que todos los jóvenes detenidos en establecimientos de la justicia penal reciban una valoración de salud mental, consumo de sustancias, factores de riesgo de suicidio y problemas emocionales y comportamentales (Penn \& Thomas, 2005). Para realizar estas tareas de evaluación y diagnóstico en adolescentes existen instrumentos clínicos ampliamente contrastados, como el sistema de evaluación multiaxial ASEBA (Achenbach, 2009) o la Entrevista Diagnóstica para Niños y Adolescentes (Shaffer, Fisher, Lucas, Hilsenroth, \& Segal, 2004). No obstante, muchos contextos institucionales, como los sistemas penales juveniles, no cuentan con los recursos necesarios para implementar estas técnicas de manera universal. Esta limitación puede solventarse empleando instrumentos de cribado que tienen como objetivo la detección rápida de jóvenes de alto riesgo o en estado de crisis que requieran intervención inmediata, así como también la identificación de aquellos que necesiten una evaluación de salud mental más exhaustiva (Grisso et al., 2005; Penner et al., 2011).

Uno de los instrumentos de cribado específicamente diseñado para el contexto penal juvenil es el Instrumento Massachusetts para el Cribado de Jóvenes (MAYSI-2; Grisso, Barnum, Fletcher, Cauffman, \& Peuschold, 2000). E1 MAYSI-2 es útil para detectar la presencia de problemas cognitivos, emocionales, conductuales y de abuso de sustancias que comporten un riesgo a corto plazo en jóvenes en conflicto con la ley penal (Grisso \& Barnum, 2006; 
Grisso et al., 2005). Ha sido traducido a 13 idiomas y administrado en múltiples estudios, con muestras que varian desde 60 a 18.000 casos, en adolescentes detenidos y condenados, con edades desde los 10 hasta los 18 años.

E1 MAYSI-2 se administra entre las 24 y 48 horas posteriores al ingreso del joven en una institución judicial y el tiempo de administración y puntuación no supera los 20 minutos. Es de muy bajo costo y no requiere experiencia clínica para ser administrado, puntuado e interpretado. Para responderlo no se requiere una escolarización superior al quinto grado (Grisso \& Barnum, 2006). Es un autoinforme de 52 items dicotómicos (sí/no) cuyas respuestas positivas se suman para obtener la puntuación en cada una de las siete subescalas. Seis de ellas evalúan eventos sucedidos en los meses previos a la administración: Uso de alcohol y drogas (UAD), Enojo-irritabilidad (EI), Depresión-ansiedad (DA), Somatización (S), Ideación suicida (IS) y Perturbación del pensamiento (PP). La subescala Experiencias traumáticas (ET) explora eventos que el joven puede haber sufrido en cualquier momento de su vida (Grisso \& Barnum, 2006). Cada subescala tiene dos puntos de corte (exceptuando ET) que se establecieron en el estudio original con muestras de Massachusetts ( $n=1279,12$ a 17 años) y California ( $n=4004,13$ a 17 años) sobre la base de las correlaciones entre las puntuaciones en el MAYSI-2 y las obtenidas en el Millon Adolescent Clinical Inventory y el Child Behavior Checklist-Youth Self Report (Grisso \& Barnum, 2006). Si la sumatoria de items presentes de cada subescala no alcanza ningún punto de corte, se dirá que el adolescente "no aplica" para dicha subescala. Si, en cambio, la sumatoria de items presentes alcanza el primer o segundo punto de corte, se dirá que el adolescente aplica para el punto de corte "precaución" o "peligro" respectivamente.

La categoría "precaución" indica la posibilidad de que el adolescente requiera atención en salud mental, mientras que la categoría "peligro" indica una puntuación excepcionalmente elevada en comparación con otros jóvenes del sistema de justicia juvenil, e identifica a aquellos adolescentes que requieren alta prioridad en la evaluación comprensiva y en la atención de necesidades de salud mental. Después de que los adolescentes responden el autoinforme, se realiza un segundo cribado a fin de explorar respuestas específicas en aquellas subescalas en las que se alcanzó algún punto de corte e identificar la necesidad de una intervención inmediata, si la hubiera (Grisso \& Barnum, 2006).

La prevalencia general de necesidades de salud mental identificada con este instrumento (es decir, puntuaciones por encima de algún punto de corte) oscila en los diferentes estudios entre el $70 \%$ y el $80 \%$, siendo las más prevalentes aquellas relacionadas al uso problemático de sustancias $(34 \%$ $80 \%)$, los síntomas somáticos $(28 \%$ - 54\%), los sentimientos de ira, frustración e irritabilidad $(28 \%-56 \%)$ y las alteraciones de pensamiento (24\% - 49\%). También se han reportado con frecuencia sintomas depresivos y ansiosos $(19 \%-43 \%)$ y pensamientos o intenciones autolesivos (13\%-18\%) (Cauffman, 2004; Gretton \& Clift, 2011; Grisso \& Barnum, 2006; McArdle \& Lambie, 2018; Stathis et al., 2008; White, Lau, \& Aalsma 2016). Cerca de la mitad de los casos evaluados en el estudio original presentaron comorbilidad entre dos o más subescalas (Grisso \& Barnum, 2006). No obstante, esta variable ha sido poco explorada en los estudios subsiguientes que emplearon el MAYSI-2. 


\section{El presente estudio}

La legislación vigente en Argentina resalta la importancia de atender y proteger los derechos de los adolescentes, y entre ellos se encuentra el derecho a la salud integral (e.g., Ley $\mathrm{N}^{\circ} 26.061,2014$; UNICEF, 2018). También las normativas internacionales destacan la importancia de una evaluación comprehensiva y basada en la evidencia de las condiciones socio-sanitarias de los jóvenes que ingresan al sistema penal juvenil (Penn \& Thomas, 2005). No obstante, tras una búsqueda en EbscoHost, Dialnet, PSICODOC, PsycINFO y Google Académico en la que se emplearon los siguientes términos y sus combinaciones: adolescentes, jóvenes, infractores, conflicto con la ley penal, detenidos, salud mental, enfermedad mental, trastorno mental, psicopatologia, necesidades; no se han encontrado artículos publicados en las últimas tres décadas que informen sobre prevalencias o técnicas para la evaluación de problemas de salud mental en la población residente en el sistema penal juvenil de Argentina. Por ello, el objetivo principal de este estudio es identificar tipos y prevalencia de necesidades de salud mental presentes en una muestra de adolescentes en conflicto con la ley penal en la provincia de Córdoba mediante un instrumento con gran aceptación internacional, aunque novedoso en el contexto de este estudio. Adicionalmente, son objetivos del estudio analizar las tasas de comorbilidad de problemas de salud mental y explorar las experiencias traumáticas y de victimización de los participantes.

\section{METODOLOGÍA}

\section{Tipo de estudio y participantes}

Se realizó un estudio descriptivo con un diseño ex post facto retrospectivo (Montero \& Leon, 2002). La selección de los participantes se llevó a cabo mediante un muestreo no probabilístico accidental. La muestra se conformó de 55 adolescentes varones que tenían entre 13 y 20 años en la instancia de admisión a una institución socioeducativa juvenil de régimen cerrado de la ciudad de Córdoba (Argentina).

La tabla 1 presenta las características de los participantes. Los delitos presuntamente cometidos fueron agrupados del siguiente modo: robo (efectivo $\mathrm{y}$ tentativo), hurto (efectivo y tentativo), violencia grave (homicidio, lesiones graves y abuso sexual) y otros (encubrimiento, venta de drogas y sin clasificar). La aprobación del estudio fue realizada por el Área de Capacitación de la Secretaría de Niñez, Adolescencia y Familia de la Provincia de Córdoba. La directora del Centro de Admisión dio, en calidad de representante legal, su asentimiento para la participación de los jóvenes. 
Tabla 1.

Características de los participantes $(n=55)$.

\begin{tabular}{lcc}
\hline & $\boldsymbol{M}$ & $\boldsymbol{D E}$ \\
\cline { 2 - 3 } Edad: $M(D E)$ & 16.42 & 1.07 \\
Numero ingresos previos: $M(D E)$ & 2.43 & 2.35 \\
& $\boldsymbol{f}$ & $\%$ \\
\cline { 2 - 3 } Nivel educativo & & \\
$\quad$ Primario incompleto & 7 & 12.7 \\
$\quad$ Primario completo & 2 & 3.6 \\
$\quad$ Secundario & 46 & 83.6 \\
Abandonó los estudios & 48 & 87.3 \\
Trabajaba antes del ingreso & 41 & 74.5 \\
Ingreso previo al centro & 23 & 41.8 \\
Consumo regular de drogas antes del ingreso & 42 & 76.4 \\
Atención en salud mental antes del ingreso & 30 & 54.5 \\
Tratamiento por consumo de drogas antes del ingreso & 16 & 29.1 \\
Causa penal que motiva el ingreso & & \\
$\quad$ Robo & 38 & 69.1 \\
Violencia grave & 6 & 10.9 \\
$\quad$ Otros & 6 & 10.9 \\
$\quad$ Hurto & 5 & 9.1 \\
\hline
\end{tabular}

\section{Instrumentos}

Se diseñó un cuestionario ad hoc para recoger información de las características sociodemográficas de los participantes (edad, nivel educativo, actividad laboral, consumo de sustancias, antecedentes delictivos y antecedentes clínicos). Inicialmente, el cuestionario fue completado por los participantes mediante autoinforme. Posteriormente, una de las coautoras revisó los archivos oficiales para contrastar y complementar la información.

Para evaluar las necesidades de salud mental se empleó una versión traducida al español del Massachusetts Youth Screening Instrument - Versión 2 (Grisso \& Barnum, 2006), cuya traducción inversa fue realizada por una traductora nativa y aprobada por el autor principal del instrumento (T. Grisso, comunicación personal, 6 de diciembre del 2018). El MAYSI-2 ha demostrado indices de consistencia interna entre aceptables y buenos en la mayoría de las subescalas $(a=.60-.90)$ (Archer, Stredny, Mason, \& Arnau, 2004; Grisso et al., 2000; Hayes, McReynolds, \& Wasserman, 2005). También se reportaron buenos indices de fiabilidad test-retest $(C C I=0.73$ a 0.89$)$ y de validez concurrente con el Millon Adolescent Clinical Inventory, el Child Behavior Checklist-YSR (Colins et al., 2014; Grisso et al., 2000; Shulman, Bechtold, Kelly, \& Cauffman 2018) y la Entrevista Diagnóstica para Niños DISC-IV (Colins, Grisso, Mulder, \& Vermeiren, 2014). Además, ha demostrado índices de sensibilidad y especificidad entre el $70 \%$ y el $90 \%$ que resultan aceptables para un instrumento de cribado (Grisso et al., 2000; Shulman et al., 2018). La validez de constructo de adaptaciones del MAYSI-2 en diversos países y muestras se ha explorado mediante análisis factoriales confirmatorios cuyos resultados apoyan las siete subescalas propuestas originalmente (Colins et al., 2014; Demirbaş-Çakır et al., 2015; Zannella, Eno Louden, Kennealy, \& Kang, 2018). En la presente muestra los indices de fiabilidad fueron buenos para las subescalas UAD e IS ( $a=.82$ y .77 , respectivamente), moderados para DA ( $a=$ 
.61) y bajos para el resto de subescalas $(a=.49-.56)$ (Tavakol \& Dennick, 2011).

\section{Procedimiento}

Se obtuvo la autorización del organismo público a cargo de la institución y de la dirección del centro de admisión, quienes controlaron que el protocolo de investigación cumpliera las normativas éticas que rigen la investigación en ciencias sociales (American Psychological Association, 2017) y actuaron como referentes institucionales para autorizar la administración del instrumento en la institución (Art. 8.01, American Psychological Association, 2017; Hoge, 2012). Una investigadora leyó la hoja de información con las consideraciones éticas del estudio de forma conjunta a los participantes, realizó preguntas de chequeo para asegurar la comprensión de toda la información y solicitó el consentimiento informado. Los datos se recogieron con el conocimiento y el consentimiento escrito de cada uno de los participantes. La información de los casos que aceptaron participar fue recogida en tres fases: revisión del expediente, entrevista semi-estructurada y administración del MAYSI-2 en un rango de 0 a 5 dias $(M=1.87$ dias; $D E=1.07)$ después del ingreso al centro socioeducativo.

\section{Análisis de datos}

El análisis de datos se realizó con la versión 24 del Paquete Estadístico para Ciencias Sociales. Se realizaron análisis descriptivos de las puntuaciones del MAYSI-2 y se calcularon las distribuciones de frecuencias y porcentajes para cada item. Se describieron la frecuencia y porcentaje de sujetos que aplica para los puntos de corte de precaución y de peligro en cada subescala. Los puntos de corte considerados en este estudio fueron los publicados en el manual del instrumento (Grisso \& Barnum, 2006). Para conocer las necesidades de salud mental con mayor probabilidad de presentarse de manera simultánea, se calculó la co-ocurrencia de puntuaciones por encima de algún punto de corte en dos o más subescalas del MAYSI-2. Es decir, si un sujeto presentaba puntuaciones por encima del punto de corte precaución o peligro en dos o más escalas se consideraba comorbilidad entre ellas. Posteriormente, se realizaron análisis de asociación bivariada mediante la conformación de tablas de contingencia y pruebas de chi cuadrado $\left(\chi^{2}\right)$ entre las diferentes subescalas, con la finalidad de contrastar la hipótesis nula de independencia entre las variables. Previo a la estimación de $\chi^{2}$, se chequeó el supuesto de número de casos por casilla, encontrando que la totalidad de las celdas de las tablas de contingencia cumplian con la condición de tener valores esperados mayores a 5 (George \& Mallery, 2016). Se emplearon también los Residuos Tipificados Corregidos (RTC) de las tablas de contingencia para interpretar la relación y el grado de asociación entre las variables estudiadas.

\section{RESULTADOS}

\section{Puntuaciones en los ítems del MAYSI-2}

En el análisis a nivel de ítems se observó que todos los ítems fueron positivos en al menos un caso, con prevalencias que oscilaron entre el $20 \%$ y el $60 \%$ (tabla 2). El más prevalente fue el referido a haber sentido 
palpitaciones en estados de nerviosismo o ansiedad. La tabla 2 muestra sobre la línea punteada los ítems que fueron positivos en más de la mitad de los encuestados. Entre ellos es posible identificar numerosos indicadores relacionados con la desregulación emocional (por ej. consumo de sustancias para afrontar estados emocionales negativos o pobre control emocional o conductual).

Tabla 2.

Porcentajes de casos con respuestas positivas en los ítems del MAYSI-2.

\begin{tabular}{|c|c|c|c|c|c|c|c|c|c|c|c|c|c|}
\hline \multicolumn{14}{|c|}{ Subescalas del MAYSI-2 } \\
\hline \multicolumn{2}{|c|}{ UAD } & \multicolumn{2}{|c|}{ EI } & \multicolumn{2}{|c|}{ DA } & \multicolumn{2}{|c|}{$\mathbf{s}$} & \multicolumn{2}{|c|}{ IS } & \multicolumn{2}{|c|}{ PP } & \multicolumn{2}{|c|}{ ET } \\
\hline Item & $\%$ & Item & $\%$ & Item & $\%$ & Item & $\%$ & Item & $\%$ & Item & $\%$ & Item & $\%$ \\
\hline $\begin{array}{l}33 \\
40 \\
10\end{array}$ & $\begin{array}{r}618 \\
50.9 \\
47.3\end{array}$ & $\begin{array}{c}8 \\
44 \\
2\end{array}$ & $\begin{array}{l}58.2 \\
52.7 \\
50.9\end{array}$ & 3 & 52.7 & $\begin{array}{l}28 \\
30 \\
27\end{array}$ & $\begin{array}{l}68.9 \\
67.3 \\
52.7\end{array}$ & & & & & $\begin{array}{l}52 \\
46\end{array}$ & $\begin{array}{c}65.5 \\
60\end{array}$ \\
\hline $\begin{array}{l}23 \\
24 \\
45 \\
19 \\
37\end{array}$ & $\begin{array}{l}43.6 \\
34.5 \\
27.3 \\
21.8 \\
21.8\end{array}$ & $\begin{array}{c}39 \\
13 \\
6 \\
42 \\
7 \\
35\end{array}$ & $\begin{array}{l}49.1 \\
45.5 \\
43.6 \\
38.2 \\
36.4 \\
34.5\end{array}$ & $\begin{array}{l}17 \\
34 \\
35 \\
41 \\
51 \\
21 \\
14 \\
47\end{array}$ & $\begin{array}{c}43.6 \\
40 \\
34.5 \\
34.5 \\
34.5 \\
32.7 \\
18.2 \\
16.4\end{array}$ & $\begin{array}{l}43 \\
31 \\
29\end{array}$ & $\begin{array}{l}45.5 \\
34.5 \\
27.3\end{array}$ & $\begin{array}{l}11 \\
16 \\
18 \\
22 \\
47\end{array}$ & $\begin{array}{l}23.6 \\
18.2 \\
18.2 \\
16.4 \\
16.4\end{array}$ & $\begin{array}{c}26 \\
20 \\
32 \\
9 \\
25\end{array}$ & $\begin{array}{c}45.5 \\
20 \\
18.2 \\
14.5 \\
3.6\end{array}$ & $\begin{array}{l}48 \\
51 \\
49\end{array}$ & $\begin{array}{c}36.4 \\
34.5 \\
20\end{array}$ \\
\hline
\end{tabular}

Nota: $n=55 ;$ UAD = Uso de alcohol y drogas, EI = Enojo-irritabilidad, DA = Depresión- ansiedad, $\mathrm{S}=$ Somatización, IS = Ideación suicida, $\mathrm{PP}=$ Perturbación del pensamiento, ET = Experiencias traumáticas. La línea punteada divide los ítems con prevalencias mayores y menores al $50 \%$.

\section{Puntuaciones en las subescalas del MAYSI-2}

Del total de los participantes, el $87.3 \%(n=48)$ obtuvo puntuaciones por encima de algún punto de corte para al menos una subescala del MAYSI- 2. Más específicamente, el $83.6 \%$ aplicó para el punto de corte de "precaución" ( $n$ $=46$ ) en al menos una subescala, y el 40\% aplicó para el punto de corte de "peligro" $(n=22)$ en al menos una subescala. La figura 1 detalla para cada subescala la proporción de casos en cada punto de corte.

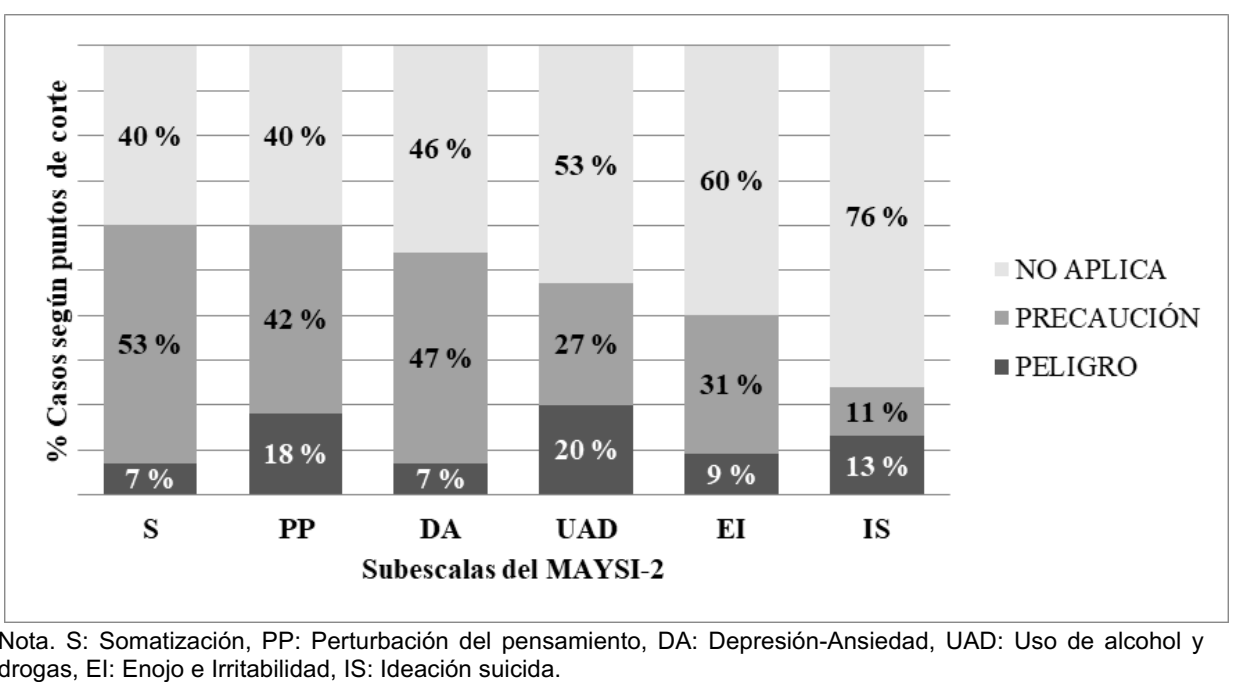

Figura 1. Proporción de casos según puntos de corte en las subescalas del MAYSI-2. 
La subescala Experiencias traumáticas no posee puntos de corte, sino que sirve para indicar la proporción de adolescentes que responden afirmativamente en algún ítem. El 87.3\% $(n=48)$ de los participantes indicó haber vivenciado al menos una experiencia traumática a lo largo de su vida y el 23\% indicó haber vivenciado más de cuatro. La media grupal de ítems positivos en esta subescala fue de $2.42(D E=1.25)$. Esto implica que, en promedio, estos jóvenes han vivenciado entre dos $y$ tres experiencias traumáticas a lo largo de su vida.

\section{Comorbilidad de necesidades de salud mental según el MAYSI-2}

El $78.2 \%$ de los adolescentes $(n=43)$ obtuvo puntuaciones por encima de un punto de corte en dos o más subescalas. Aproximadamente un cuarto de estos casos $(n=10)$ lo hizo en 5 o 6 subescalas. La tabla 3 muestra las frecuencias absolutas y relativas de casos que puntuaron por encima de algún punto de corte para cada par de subescalas y los Residuos Tipificados Corregidos (RTC) de las asociaciones. Se encontraron múltiples asociaciones entre las diferentes subescalas, lo que implica que si el sujeto puntuó por encima del punto de corte precaución o peligro para una subescala es probable que también puntúe por encima del punto de corte precaución o peligro para la otra (tabla 3). En este estudio lo expuesto se considera un indicador de comorbilidad entre ambas condiciones. Los RTC de las asociaciones estadísticamente significativas a un nivel de significación del 5\% oscilaron entre 2.1 y 3.5. Las condiciones que se presentaron conjuntamente en niveles de precaución o peligro en una mayor proporción de casos fueron las definidas por las subescalas de somatización y de perturbación del pensamiento $(R T C=3.5)$.

Tabla 3.

Frecuencias absolutas y relativas de casos con puntuaciones por encima de algún punto de corte en pares de subescalas del MAYSI-2.

\begin{tabular}{|c|c|c|c|c|c|}
\hline & EI & DA & $\mathbf{S}$ & IS & PP \\
\hline Subescalas & $f(\% ; R T C)$ & $f(\% ; R T C)$ & $f(\% ; R T C)$ & $f(\% ; R T C)$ & $f(\% ; R T C)$ \\
\hline UAD & $13(23.6 ; 1.4)$ & $15(27.3 ; 0.4)$ & $17(30.9 ; 0.8)$ & $9(16.4 ; 1.8)$ & $16(29.1 ; 0.2)$ \\
\hline EI & - & $17(30.9 ; 2.8)^{\star *}$ & $17(30.9 ; 2.1)^{\star}$ & $7(12.7 ; 1.2)$ & $17(30.9 ; 2.1)^{*}$ \\
\hline DA & - & - & $22(40.0 ; 2.2)^{*}$ & $11(20.0 ; 2.5)^{*}$ & $22(40.0: 2.2)^{*}$ \\
\hline S & - & - & - & $10(36.4 ; 1.4)$ & $26(47.3 ; 3.5)^{\star \star *}$ \\
\hline IS & - & - & - & - & $11(20.0 ; 2.1)^{*}$ \\
\hline
\end{tabular}

Nota: Valor de $p$ para $x^{2:}{ }^{*} p<.05 ;{ }^{* *} p<.01 ;{ }^{* * *} p<.001 ; R T C=$ Residuos Tipificados Corregidos. UAD: Uso de alcohol drogas, El: Enojo-irritabilidad, DA: Depresión-ansiedad, S: Somatización, IS: Ideación suicida, PP: Perturbación del pensamiento, ET: Experiencias traumáticas.

\section{DISCUSIÓN}

Las necesidades de salud mental parecen estar presentes en proporciones alarmantes en los adolescentes cuyo comportamiento antinormativo alcanza formas delictivas (Bailey et al., 2006; Fazel et al., 2008; Penner et al., 2011). Ante el interrogante sobre si esto era un hecho contrastable también en el contexto de este estudio, se administró el MAYSI-2 a una muestra de 55 adolescentes ingresados en una institución penal juvenil cerrada.

Este estudio aporta evidencia local sobre la gran cantidad de adolescentes privados de libertad que requieren atención específica $y$ 
especializada para atender sus padecimientos mentales. Un $90 \%$ de los adolescentes evaluados presentaron puntuaciones que indicaban la posible presencia de necesidades de salud mental. Esta proporción resultó más elevada que la encontrada en estudios previos que emplean el mismo instrumento (Cauffman, 2004; Gretton \& Clift, 2011; Grisso \& Barnum, 2006; McArdle \& Lambie, 2018; Stathis et al., 2008; White et al., 2016). Otros estudios que emplean instrumentos para evaluaciones en profundidad o los criterios diagnósticos de manuales internacionales reportan prevalencias de necesidades de salud mental que rondan el 70\% (Colins et al., 2010), y llegan a superar el $80 \%$ en regiones cercanas al presente estudio (Gaete et al., 2014).

Aunque existe un acuerdo general acerca de la amplia proporción de adolescentes involucrados en el sistema penal que presentan problemas de salud mental (Penner et al., 2011), no está claro qué factores podrían explicar la variabilidad entre estudios en las tasas reportadas. Una posible explicación es que los países con sistemas sanitarios y educativos fuertes y basados en políticas públicas eficaces sean capaces de atender las necesidades de salud de sus ciudadanos en estadios vitales tempranos y así detener su evolución hacia estadios más tardíos (Organización Mundial de la Salud, 2008). Numerosos programas de intervención temprana sobre familias y niños han demostrado eficacia en la prevención del desarrollo futuro de eventos vitales negativos como los problemas de salud mental y la delincuencia (Dodge et al., 2015). Por el contrario, contextos menos protegidos generarán mayor vulnerabilidad para dichos eventos (Wolf, Gray, \& Fazel 2014). A su vez, estos eventos tenderán a correlacionar entre sí, de modo que si un joven comete un delito es más probable que presente necesidades de salud mental comparado con otro que no haya cometido un acto delictivo (Vermeiren, 2003). Este efecto de potenciación recíproca entre factores de riesgo representa un verdadero desafio a los sistemas penales juveniles (Underwood \& Washington, 2016).

También diferencias metodológicas podrían explicar las variaciones entre estudios de las tasas de prevalencia de los problemas de salud mental de los jóvenes (Penner et al., 2011). Por ejemplo, se encontrarán cifras más bajas de estos problemas cuando los estudios evalúen esta variable mediante definiciones operacionales más restrictivas (diagnóstico de trastorno mental específico en contraposición a presencia de síntomas), cuando su evaluación comprenda períodos más acotados (últimos meses en contraposición a toda la vida) o cuando se estudien poblaciones en menor riesgo (jóvenes en la comunidad o en medio abierto en contraposición a jóvenes sentenciados o privados de libertad).

Otro interrogante de este estudio estuvo relacionado con la comorbilidad de las condiciones evaluadas. Nuestras cifras, que indicaron que 8 de cada 10 adolescentes podrian presentar dos o más tipos de necesidades de salud mental, fueron similares a las de investigaciones previas (Abram et al., 2015; Schufelt \& Cocozza, 2006), e indican aproximadamente un 30\% más de adolescentes con comorbilidad que en el estudio original con el MAYSI2 (Grisso et al., 2000). La elevada comorbilidad entre diagnósticos es un hecho identificado en el ámbito de la psicopatología y se encuentra en la base de la crítica a los sistemas diagnósticos categoriales, en contraposición a los dimensionales y transdiagnósticos (Pérez-Álvarez, 2019). Nuestros hallazgos soportan la idea de que la evaluación psicológica, especialmente en los procesos de admisión, debe orientarse de manera amplia y comprensiva al 
análisis del repertorio conductual, emocional y cognitivo de las personas y sus déficits.

En contraste con estudios previos (Abram et al., 2015; Schufelt \& Cocozza, 2006), en la muestra estudiada la somatización y las alteraciones de pensamiento fueron las necesidades comórbidas más frecuentes. Ambas condiciones podrian estar asociadas a transacciones estresantes previas e inmediatas al ingreso en la institución. La ansiedad y sus diversas facetas (fisiológica, cognitiva y emocional) constituyen una respuesta definida como mecanismo de afrontamiento al estrés (Lazarus \& Folkman, 1984). De hecho, en un segundo cribado exploramos los motivos de las respuestas positivas en las subescalas donde el adolescente alcanzó algún punto de corte y advertimos que diversos sintomas (por ej. inquietud o hiperactividad [ $n=12$ ], dificultades de sueño $[n=11]$ y síntomas de depresión o ansiedad $[n=7])$ surgieron después del ingreso al centro de internamiento. Este hallazgo destaca la importancia de realizar un seguimiento a los adolescentes durante su estancia en el establecimiento, a fin de identificar el surgimiento de nuevas necesidades o riesgos derivados del contexto de encierro (Penn \& Thomas, 2005).

Aunque el abuso de sustancias se ha establecido de manera sólida como un factor de riesgo de la conducta delictiva en adolescentes (Colins et al., 2011), es probable que este estudio no haya captado a la población de adolescentes con trastornos graves por consumo de sustancias que en este contexto suelen ser derivados a servicios especializados. No obstante, la subescala de Uso de alcohol y drogas fue una de las más prevalentes sobre el punto de corte de "peligro". Algo similar se reportó en el estudio original de California (Grisso et al., 2000). De modo general, estos hallazgos indican que, aunque no es la condición que más acompaña a las otras, es la que se presenta en niveles de mayor gravedad y que, por lo tanto, requiere una intervención urgente, intensiva y especializada (Belenko et al., 2017).

Además de las condiciones clínicas previamente mencionadas, también se reportó un número significativo de experiencias traumáticas y experiencias de victimización a lo largo de la vida de los participantes. Esto resultó coincidente con estudios previos (Abram et al., 2004; Ford et al., 2008) que demuestran que las experiencias traumáticas no son algo excepcional en la vida de estos jóvenes. En este estudio, la experiencia traumática más frecuente fue haber presenciado a alguien gravemente herido o muerto. Esto podría explicarse por el hecho de que la violencia urbana es un fenómeno que afecta especialmente a los jóvenes con un perfil sociodemográfico similar al de esta muestra, es decir, residentes en barrios de vulnerabilidad social y con escasos recursos económicos (UNODC, 2019). Así, por la rutina habitual de los jóvenes, que conlleva un mayor tiempo con sus pares en entornos con altas oportunidades criminógenas, tendrian mayor probabilidad de presenciar (y de sufrir) estos eventos (Henson, Wilcox, Reyns, \& Cullen, 2010).

Uno de cuatro jóvenes evaluados en este estudio presentó puntuaciones en los items de suicidalidad más elevadas que las esperables sobre la base de estudios previos (Stokes, McCoy, Abram, Byck, \& Teplin, 2015). La mayoría de ellos presentaron, además, niveles elevados de sintomas ansiosos y depresivos que suelen ser sólidos predictores de la conducta suicida (Scott et al., 2015). Estos resultados, junto con los antecedentes que demuestran un mayor riesgo de conducta suicida en los adolescentes en el sistema de justicia penal comparados con los de población general (Hayes, 2009), indican una clara 
necesidad de identificar su riesgo tempranamente y actuar en consecuencia (Stokes et al., 2015).

En relación con la atención recibida por razones de salud mental descrita en la tabla 1, más de la mitad de los participantes la habian recibido antes del ingreso actual, y un tercio la recibió por problemas asociados al consumo de sustancias. Estas cifras concuerdan con resultados de otros estudios que indican que, aunque la mayoria de los problemas de salud mental de los adolescentes en conflicto con la ley penal se detectan tras el ingreso al sistema de justicia, su origen suele ser previo a esa instancia (Hammersley, Marsland, \& Reid, 2003). No obstante, en un análisis complementario, encontramos que los adolescentes con ingresos previos (reincidentes) estaban sobrerrepresentados entre quienes reportaban haber recibido atención psicológica o psiquiátrica previa, algo que también ha sido descrito en estudios previos (White et al., 2016). Por lo tanto, es probable que la asistencia previa reportada se haya recibido dentro de la institución en ingresos previos, más que en servicios comunitarios que suelen ser de dificil acceso en estos casos (UNICEF, 2016). De hecho, literatura previa indica que menos de un tercio de los adolescentes reporta contactos previos con un servicio de salud mental externo a la institución (Hammersley et al., 2003).

\section{Limitaciones del presente estudio}

Algunas limitaciones del presente trabajo deben tenerse en cuenta al momento de interpretar los resultados. En primer lugar, el MAYSI-2 aún no cuenta con una adaptación psicométrica local. En este estudio se realizó la traducción (directa e inversa) y se calcularon los indices de consistencia interna de las subescalas. Algunas de ellas (Perturbación del pensamiento, Somatización y Enojo-irritabilidad) presentaron valores bajos de consistencia interna en esta muestra. Por lo tanto, su consideración como indicadores de un constructo latente puede ser cuestionada. No obstante, esto no invalida la utilidad clínica de cada indicador. Los análisis a nivel de items permitieron identificar sintomas especificos que efectivamente fueron reportados como presentes. Incluso un tercio de ellos fue reportado por más de la mitad de la muestra.

Por otra parte, la muestra fue accidental y, por lo tanto, no es representativa de la totalidad de la población juvenil en contexto de encierro en Argentina. En consecuencia, los resultados informan sobre este grupo particular de sujetos. No obstante, las características de los participantes de este estudio no difieren de las reportadas para otras muestras de adolescentes ingresados en la misma institución con relación al promedio de edad, el nivel educativo, el núcleo de convivencia, los delitos más prevalentes, la presencia de antecedentes delictivos familiares, la historia de abuso de sustancias y la proporción de reincidentes (Orpianesi, 2018; Secretaría de Niñez, Adolescencia y Familia, 2017). A pesar de que permanezca como una pregunta empírica a ser testeada, no sería improbable que los resultados de nuestro estudio pudieran generalizarse más allá de los participantes de este estudio.

\section{Futuras líneas de investigación}

Este estudio abre una línea de investigación novedosa en el contexto local. Futuros estudios realizarian un provechoso aporte al contrastar las propiedades psicométricas del MAYSI-2 en muestras representativas del ámbito penal juvenil local, no solo en el medio cerrado sino también en los 
dispositivos de medio abierto. Además de explorar su validez convergente con otros instrumentos diagnósticos y su validez predictiva en relación con criterios externos (por ej. internaciones), es importante revisar los puntajes de corte y diseñar manuales de procedimientos adecuados a cada contexto particular (Grisso et al., 2005). Esto no solo favorece el desarrollo de la investigación comparada con los hallazgos de otros países, sino también la implementación y aceptación del instrumento entre los profesionales de la salud que trabajan en el contexto penal juvenil local (Tovar, 2007).

Dado que estudios previos indican que las adolescentes mujeres en conflicto con la ley presentan una mayor prevalencia de necesidades de salud mental que los varones (Fazel et al., 2008; Grisso \& Barnum, 2006), resultan prometedores estudios con el MAYSI-2 en esta población. Nuevamente, la comparación de estos resultados con los propios de otros contextos (Archer et al., 2004; Grisso et al., 2000; Hayes et al., 2005; Vincent, Grisso, Terry, \& Banks, 2008), mejorará el entendimiento de las particularidades de esta población que permanece inexplorada en el contexto local.

\section{Conclusión}

Es posible que, en muchos de los casos, la institucionalización se convierta en la única posibilidad de los jóvenes de acceder a los servicios de salud mental. En consecuencia, implementar una estrategia de cribado de las diferentes necesidades de salud mental en la admisión a los centros correccionales juveniles es altamente recomendable. Esto facilitaría la progresión de los casos hacia las instancias de evaluación, intervención y seguimiento adecuadas a cada uno. Esta recomendación se fundamenta en la amplia proporción de jóvenes que presenta dificultades en el área de la salud mental, el riesgo que estas dificultades representan para ellos mismos o para terceros y la influencia que pueden tener en la actividad delictiva. Para garantizar la implementación y sostenibilidad en el tiempo de esta estrategia, es necesario que las autoridades judiciales reconozcan estos protocolos como herramientas ineludibles para la toma de decisiones profesionales al asignar recursos a un caso (Bailey et al., 2006; Grisso et al., 2005).

La cifra de adolescentes privados de libertad, que en Argentina ronda los 4000 individuos (UNICEF, 2015), no refleja el carácter de excepcionalidad que las normas internacionales asignan a esta medida (Secretaría de Derechos Humanos, 2010). No obstante, si las politicas de prevención del delito en jóvenes se basaran en la evidencia disponible sobre la eficacia limitada de la privación de libertad para la reinserción de los adolescentes (Wilson \& Hoge, 2013), sería esperable que estas cifras se redujeran. Hasta que eso ocurra, e incluso después, las técnicas de cribado se constituyen como una opción útil y específica en el cumplimiento de la valoración inmediata y universal de las necesidades de salud mental en el contexto de la justicia penal juvenil (Grisso et al., 2000).

\section{REFERENCIAS}

Abram, K. M., Teplin, L. A., Charles, D. R., Longworth, S. L., McClelland, G. M., \& Dulcan, M. K. (2004). Posttraumatic Stress Disorder and trauma in youth in juvenile detention. Archives of General Psychiatry, 61(4), 403. https://doi.org/10.1001/archpsyc.61.4.403

Abram, K. M., Zwecker, N. A., Welty, L. J., Hershfield, J. A., Dulcan, M. K., \& Teplin, L. A. (2015). Comorbidity and continuity of psychiatric disorders in youth after detention. JAMA Psychiatry, 72(1), 84. https://doi.org/10.1001/jamapsychiatry.2014.1375

Achenbach, T. M. (2009). Achenbach system of empirically based assessment (ASEBA): Development, findings, theory, and applications. Vermont: University of Vermont, Research Center of Children, Youth 
\& Families.

American Psychological Association (2017). Ethical principles of psychologists and code of conduct. Code of conduct. Washington: American Psychological Association.

Archer, R. P., Stredny, R. V., Mason, J. A., \& Arnau, R. C. (2004). An examination and replication of the psychometric properties of the Massachusetts Youth Screening Instrument - Second Edition (MAYSI-2) among adolescents in detention settings. Assessment, 11(4), 290-302. https://doi.org/10.1177/1073191104269863

Bailey, S., Doreleijers, T., \& Tarbuck, P. (2006). Recent developments in mental health screening and assessment in juvenile justice systems. Child and Adolescent Psychiatric Clinics of North America, 15(2), 391-406. https://doi.org/10.1016/j.chc.2005.12.004

Belenko, S., Knight, D., Wasserman, G. A., Dennis, M. L., Wiley, T., Taxman, F., ... Sales, J. (2017). The juvenile justice behavioral health services cascade: A new framework for measuring unmet substance use treatment services needs among adolescent offenders. Journal of Substance Abuse Treatment, 74, 80-91. https://doi.org/10.1016/j.jsat.2016.12.012

Bhatta, M. P., Jefferis, E., Kavadas, A., Alemagno, S. A., \& Shaffer-King, P. (2014). Suicidal behaviors among adolescents in juvenile detention: Role of adverse life experiences. PLoS ONE, 9(2), e89408. https://doi.org/10.1371/journal.pone.0089408

Cauffman, E. (2004). A statewide screening of mental health symptoms among juvenile offenders in detention. Journal of the American Academy of Child \& Adolescent Psychiatry, 43(4), 430-439. https://doi.org/10.1097/00004583-200404000-00009

Colins, O., Grisso, T., Mulder, E., \& Vermeiren, R. (2014). The relation of standardized mental health screening and categorical assessment in detained male adolescents. European Child and Adolescent Psychiatry, 24(3), 339-349. https://doi.org/10.1007/s00787-014-0584-1

Colins, O., Grisso, T., Vahl, P., Guy, L., Mulder, E., Hornby, N., Pronk, C., Markus, M., Doreleijers, T., \& Vermeiren, R. (2014). Standardized screening for mental health needs of detained youths from various ethnic origins: The Dutch Massachusetts Youth Screening Instrument-second version (MAYSI-2). Journal of Psychopathology and Behavioral Assessment, 37(3), 481-492. https://doi.org/10.1007/s10862-014-9476-4

Colins, O., Vermeiren, R., Vahl, P., Markus, M., Broekaert, E., \& Doreleijers, T. (2011). Psychiatric disorder in detained male adolescents as risk factor for serious recidivism. Canadian Journal of Psychiatry, 56(1), 44-50. https://doi.org/10.1177/070674371105600108

Colins, O., Vermeiren, R., Vreugdenhil, C. W., Doreleijers, T., \& Broekaert, E. (2010). Psychiatric disorders in detained male adolescents: a systematic literature review. Canadian Journal of Psychiatry, 55(4), 255-263. https://doi.org/10.1177/070674371005500409

De La Barra, M. F., Vicente, P. B., Saldivia, B. S., \& Melipillán, A. R. (2012). Estudio de epidemiología psiquiátrica en niños y adolescentes en Chile. Estado actual. Revista Médica Clínica Las Condes, 23(5), 521-529. https://doi.org/10.1016/S0716-8640(12)70346-2

Demirbas-Cakır, E., Sișmanlar, S. G., Cakır, U., Duymaz, M. K., Karakaya, I., Coskun, A., \& Ağaoğlu, B. (2015). Adolescent delinquency and psychopathology: Is MAYSI-2 a valid instrument to assess psychopathology risk among Turkish adolescents? Acta Medica Anatolia, 3(4), 119. https://doi.org/10.15824/actamedica.43882

Dodge, K. A., Bierman, K. L., Coie, J. D., Greenberg, M. T., Lochman, J. E., McMahon, R. J., \& Pinderhughes, E. E. (2015). Impact of early intervention on psychopathology, crime, and well-being at age 25. American Journal of Psychiatry, 172(1), 59-70. https://doi.org/10.1176/appi.ajp.2014.13060786

Fazel, S., Doll, H., \& Långström, N. (2008). Mental disorders among adolescents in juvenile detention and correctional facilities: A systematic review and metaregression analysis of 25 surveys. Journal of the American Academy of Child \& Adolescent Psychiatry, 47(9), 1010-1019. https://doi.org/10.1097/CHI.ObO13e31817eecf3

Finkelhor, D., Ormrod, R. K., \& Turner, H. A. (2007). Poly-victimization: A neglected component in child victimization. Child Abuse and Neglect, 31(1), 7-26. https://doi.org/10.1016/j.chiabu.2006.06.008

Ford, J. D., Hartman, J. K., Hawke, J., \& Chapman, J. F. (2008). Traumatic victimization, posttraumatic stress disorder, suicidal ideation, and substance abuse risk among juvenile justice-involved youth. Journal of Child \& Adolescent Trauma, 1(1), 75-92. https://doi.org/10.1080/19361520801934456

Gaete, J., Labbé, N., del Villar, P., Allende, C., \& Valenzuela, E. (2014). Prevalencia de trastornos psiquiátricos en adolescentes infractores de ley en Chile. Revista Médica de Chile, 142(11), 1377-1384. https://doi.org/10.4067/S0034-98872014001100003

George, D., \& Mallery, P. (2016). IBM SPSS statistics 23 step by step: A simple guide and reference. Londers: Routledge. https://doi.org/10.4324/9781315545899

Gretton, H. M., \& Clift, R. J. W. (2011). The mental health needs of incarcerated youth in British Columbia, Canada. International Journal of Law and Psychiatry, 34(2), 109-115. https://doi.org/10.1016/j.ijlp.2011.02.004

Grisso, T., \& Barnum, R. (2006). Massachusetts Youth Screening Instrument-Version 2: User's manual and technical report. Florida: Professional Resource Press.

Grisso, T., Barnum, R., Fletcher, K., Cauffman, E., \& Peuschold, D. (2000). Massachusetts Youth Screening Instrument for mental health needs of juvenile justice youths. Journal of The American Academy of Child and Adolescent Psychiatry, 4O(5), 541-548. https://doi.org/10.1097/00004583-20010500000013

Grisso, T.; Barnum, R.; Arbach, K. \& Orpianessi, C. (2018). Massachusetts Youth Screening Instrument Version 2: Traducción de ítems para Argentina. Manuscrito no publicado.

Grisso, T., Vincent, G., \& Seagrave, D. (2005). Mental Health Screening and Assessment in Juvenile Justice. 
New York: The Guilford Press.

Hammersley, R., Marsland, L., \& Reid, M. (2003). Substance use by young offenders: the impact of the normalisation of drug use in the early years of the 21st century. London: Home Office Research Studies. https://doi.org/10.1037/e454962008-001

Hayes, L. (2009). Juvenile suicide in confinement: A national survey. Washington: Office of Juvenile Justice and Delinquency Prevention.

Hayes, M., McReynolds, L. S., \& Wasserman, G. A. (2005). Paper and voice MAYSI-2: Format comparability and concordance with the voice DISC-IV. Assessment, 12(4), 395-403. https://doi.org/10.1177/1073191105280359

Henson, B., Wilcox, P., Reyns, B. W., \& Cullen, F. T. (2010). Gender, adolescent lifestyles, and violent victimization: Implications for Routine Activity Theory. Victims \& Offenders, 5(4), 303-328. https://doi.org/10.1080/15564886.2010.509651

Hoge, R. D. (2012). Forensic assessments of juveniles: Practice and legal considerations. Criminal Justice and Behavior, 39(9), 1255-1270. https://doi.org/10.1177/0093854812444024

Lazarus, R. S. \& Folkman, S. (1984). Stress, appraisal, and coping. New York: Springer.

Ley $\mathrm{N}^{\circ}$ 26.061. (2014). Ley de Protección integral de los derechos de niñas, niños y adolescentes. In Boletín Oficial de la Nación Argentina. Ministerio de Justicia y Derechos Humanos, Presidencia de la Nación Argentina. Recuperado de http://www.jus.gob.ar/media/3108870/ley_26061_proteccion_de_ni_os.pdf

Livanou, M., Furtado, V., Winsper, C., Silvester, A., \& Singh, S. P. (2019). Prevalence of mental disorders and symptoms among incarcerated youth: A meta-analysis of 30 studies. International Journal of Forensic Mental Health, 18(4), 400-414. https://doi.org/10.1080/14999013.2019.1619636

McArdle, S. \& Lambie, I. (2018). Screening for mental health needs of New Zealand youth in secure care facilities using the MAYSI-2. Criminal Behaviour and Mental Health, 28(3), 239-254. https://doi.org/10.1002/cbm.2067

Montero, I. \& Leon, O. (2002). Clasificación y descripción de las metodologias de investigación en Psicología. Revista Internacional de Psicología Clinica y de La Salud, 2(3), 503-508.

Moore, E., Gaskin, C., \& Indig, D. (2015). Attempted suicide, self-harm, and psychological disorder among young offenders in custody. Journal of Correctional Health Care, 21(3), 243-254. https://doi.org/10.1177/1078345815584849

Organización Mundial de la Salud. (2008). La atención primaria de salud, más necesaria que nunca. Informe sobre la salud en el mundo 2008. Recuperado de https://www.who.int/whr/2008/08_report_es.pdf

Orpianesi, C. (2018). Perfil de los adolescentes en conflicto con la ley penal en la provincia de Córdoba. Informe técnico. Córdoba: Facultad de Psicología, Universidad Nacional de Córdoba.

Penn, J. V. \& Thomas, C. (2005). Practice parameter for the assessment and treatment of youth in juvenile detention and correctional facilities. Journal of the American Academy of Child and Adolescent Psychiatry, 44(10), 1085-1098. https://doi.org/10.1097/01.chi.0000175325.14481.21

Penner, E. K., Roesch, R., \& Viljoen, J. L. (2011). Young offenders in custody: An international comparison of mental health services. International Journal of Forensic Mental Health, 10(3), 215-232. https://doi.org/10.1080/14999013.2011.598427

Pérez-Álvarez, M. (2019). Diagnóstico más allá de los sintomas: un enfoque centrado en el mundo de la vida de las personas. Cuadernos de Psiquiatría Comunitaria, 16(1), 22-38.

Schubert, C. A., Mulvey, E. P., \& Glasheen, C. (2011). Influence of mental health and substance use problems and criminogenic risk on outcomes in serious juvenile offenders. Journal of the American Academy of Child and Adolescent Psychiatry, 50(9), 925-937. https://doi.org/10.1016/j.jaac.2011.06.006

Schufelt, J. \& Cocozza, J. (2006). Youth with mental health disorders in the juvenile justice system: Results from a multi-state prevalence study. NCMHJJ Research and Program Brief. Recuperado de https://www.unicef.org/tdad/usmentalhealthprevalence06(1).pdf

Scott, M., Underwood, M., \& Lamis, D. A. (2015). Suicide and related-behavior among youth involved in the Juvenile Justice System. Child and Adolescent Social Work Journal, 32(6), 517-527. https://doi.org/10.1007/s10560-015-0390-8

Secretaria de Derechos Humanos. (2010). Justicia restaurativa en el sistema de responsabilidad penal juvenil: conceptos, perspectivas y mecanismos procesales para su implementación. Buenos Aires: Ministerio de Justicia, Seguridad y Derechos Humanos de la República Argentina.

Secretaría de Niñez, Adolescencia y Familia (2017). Adolescentes ingresados al centro de admisión y diagnóstico del Complejo Esperanza por presunta infracción de la ley penal. Protocolo de actuación. Córdoba: Secretaria de Niñez, Adolescencia y Familia, Gobierno de la Provincia de Córdoba.

Shaffer, D., Fisher, P., Lucas, C., Hilsenroth, M. J., \& Segal, D. L. (2004). The diagnostic interview schedule for children (DISC). En M. J. Hilsenroth \& D. L. Segal (Eds.), Comprehensive Handbook of Psychological Assessment (2da ed., pp. 256-270). New Jersey: John Wiley \& Sons Inc.

Shulman, E. P., Bechtold, J., Kelly, E. L., \& Cauffman, E. (2018). Mental health screening in juvenile justice settings: Evaluating the utility of the Massachusetts Youth Screening Instrument, version 2. Criminal Justice Policy Review, 29(8), 849-872. https://doi.org/10.1177/0887403416650250

Stathis, S., Letters, P., Doolan, I., Fleming, R., Heath, K., Arnett, A., \& Cory, S. (2008). Use of the Massachusetts Youth Screening Instrument to assess mental health problems in young people within an Australian youth detention center. Journal of Pediatrics and Child Health, 44(7-8), 438-443. https://doi.org/10.1111/j.1440-1754.2008.01324.x

Stokes, M. L., McCoy, K. P., Abram, K. M., Byck, G. R., \& Teplin, L. A. (2015). Suicidal ideation and behavior in youth in the juvenile Justice system: A review of the literature. Journal of Correctional Health Care, 21(3), 222-242. https://doi.org/10.1177/1078345815587001

Suárez-Soto, E., Pereda, N., \& Guilera, G. (2018). ¿Víctimas o delincuentes? Jóvenes implicados en los 
sistemas de protección y justicia juvenil en Cataluña: Un estudio exploratorio. Revista de Victimología, 8, 103-130.

Tavakol, M. \& Dennick, R. (2011). Making sense of Cronbach's alpha. International Journal of Medical Education, 2(1), 53-55. https://doi.org/10.5116/ijme.4dfb.8dfd

Tovar, J. (2007). Psicometria: Tests psicométricos, confiabilidad y validez. En A. Quintana \& W. Montgomery (Eds.), Psicología: Tópicos de actualidad (pp. 85-108). Lima: Universidad Nacional Mayor de San Marcos.

Tribunal Superior de Justicia. (2016). Adolescentes privados de la libertad conforme al procedimiento penal juvenil por presunta infracción de la ley penal en Córdoba Capital. Recuperado de http://www.justiciacordoba.gov.ar/cepj/_Estudios/Estudios.aspx

Underwood, L. A. \& Washington, A. (2016). Mental illness and juvenile offenders. International Journal of Environmental Research and Public Health, 13(2), 1-14. https://doi.org/10.3390/ijerph13020228

UNICEF. (2015). Relevamiento nacional sobre adolescentes en conflicto con la ley penal. Informe anual. Fondo de las Naciones Unidas para la Infancia. Recuperado de https://www.unicef.org/argentina/informes/relevamiento-nacional-sobre-adolescentes-en-conflictocon-la-ley-penal

UNICEF. (2016). Estado de la situación de la niñez y la adolescencia en Argentina. Fondo de las Naciones Unidas para la Infancia. https://www.unicef.org/argentina/media/2211/file/SITAN.pdf

UNICEF. (2018). Las voces de las y los adolescentes privados de libertad en Argentina. Centro de Estudios de Población. Fondo de las Naciones Unidas para la Infancia. Recuperado de https://www.unicef.org/argentina/media/3936/file

UNODC. (2019). Killing of children and young adults. Global Study on Homicide. Oficina de drogas y delito de las Naciones Unidas. Recuperado de https://www.unodc.org/unodc/en/data-and-analysis/globalstudy-on-homicide.html

Vermeiren, R. (2003). Psychopathology and delinquency in adolescents: A descriptive and developmental perspective. Clinical Psychology Review, 23(2), 277-318. https://doi.org/10.1016/S02727358(02)00227-1

Vincent, G. M., Grisso, T., Terry, A., \& Banks, S. (2008). Sex and race differences in mental health symptoms in Juvenile Justice: The MAYSI-2 national meta-analysis. Journal of the American Academy of Child \& Adolescent Psychiatry, 47(3), 282-290. https://doi.org/10.1097/CHI.0b013e318160d516

White, L., Lau, K., \& Aalsma, M. (2016). Detained adolescents: Mental health needs, treatment use, and recidivism. Journal of the American Academy of Psychiatry and the Law, 44(2), 200-212.

Wilson, H. A. \& Hoge, R. D. (2013). The effect of youth diversion programs on recidivism: A meta-analytic review. Criminal Justice and Behavior, 4O(5), 497-518. https://doi.org/10.1177/0093854812451089

Wolf, A., Gray, R., \& Fazel, S. (2014). Violence as a public health problem: An ecological study of 169 $\begin{array}{lllll}\text { countries. Social } & \text { Science }\end{array}$ https://doi.org/10.1016/j.socscimed.2013.12.006

Zannella, L., Eno Louden, J., Kennealy, P., \& Kang, T. (2018). Psychometric properties of the MAYSI-2 among Latino adolescent offenders. Criminal Justice and Behavior, 45(8), 1252-1268. https://doi.org/10.1177/0093854818774380

Recibido 21-08-2020 | Aceptado 27-11-2020 